\title{
Numerical Modeling of Fatigue Damage and Fissure Propagation under Cyclic Loadings
}

\author{
F. Bogard, * P. Lestriez and Y. Q. Guo \\ Group of Mechanics, Materials \& Structures (EA2617) \\ Université de Reims Champagne Ardenne, Moulin de la House BP1039 \\ 51687 Reims Cedex2, France
}

\begin{abstract}
The purpose of this work is to develop a numerical simulation procedure in order to predict the evolution of the fatigue damage and rupture in mechanical parts (such as rolling bearings and gears) under cyclic loadings. The study of the fatigue damage evolution, from the first defect appearance until the part's failure, is primordial in view of the preventive maintenance. The numerical procedure is based on the continuum damage mechanics and the thermodynamics of irreversible processes. The damage effects are fully coupled with the elasto-plastic constitutive laws on a macroscopic point of view. The Sines fatigue criterion for multiaxial stress states is used to estimate the lifetime of mechanical parts in terms of number of cycles. This numerical model is implemented into Abaqus/Explicit using an user's subroutine (Vumat). A cycle jumping algorithm allows to largely reduce the computation time. Some remeshing techniques are used to follow up the damage and rupture evolutions. The birth and the growth of the damage and rupture can be visualized via the element deleting and remeshing. These numerical tools are applied to a $2 \mathrm{D}$ specimen under a cyclic stretching.
\end{abstract}

KEY WORDS: fatigue damage, plasticity, rupture, cycle jumping algorithm, remeshing, lifetime.

\section{INTRODUCTION}

$\mathbf{M}$

ANUFACTURING COMPANIES ALWAYS search the means to find the defects of their machines and to determine the maintenance date in

*Author to whom correspondence should be addressed. E-mail: fabien.bogard@univ-reims.fr Figures 1-6 appear in color online: http://ijd.sagepub.com

International Journal of DAMAge MeCHANICS, Vol. 17-March 2008 
order to replace the damaged parts before their failure. The vibration analysis is a powerful tool to locate the defects (Bogard et al., 2002), but it does not allow to follow up their evolution neither to estimate the remaining lifetime of a damaged part.

In a mechanical part under cyclic loadings such as the bearings or gears, the fatigue damage may create micro-fissures, whose growth and propagation will lead to macro-fissures until the rupture of the part, even if the maximal stress is inferior to the elastic limit. To study this phenomenon, Chaboche (1974) have extended the fatigue damage model developed by Kachanov (1986) and Rabotnov (1969) to consider the fact that the damage influence on the rupture propagation velocity is greater than that of the global mechanical behavior.

The fatigue damage model of Lemaitre \& Chaboche is based on the macroscopic damage phenomena and applicable to many types of loadings, especially to cyclic loadings. The effects of the average stress, the stress amplitude, and the nonlinear cumuli of the damage (such as the effect of loading sequence, the order of several types of cycles...) are also included in the model. The tensorial character of a 3D stress state is considered by using the octahedral shear stress. The initiation of the micro-fissures and their propagation until the final rupture are represented by a continuous evolution of the damage value from 0 (virgin material) to 1 (entirely damaged material).

Many works were presented on the crack modeling by analytical approaches or finite element methods, using an elasto-plastic behavior model, a given fatigue criterion or/and a crack theory to simulate the crack initiation and its propagation. A FE-modeling in elasticity or elastoplasticity was often used to obtain the stress state under contact; then a fatigue criterion was employed to quantify the fatigue effects from the evaluated stresses and strains. A combination of a multiaxial low-cycle fatigue criterion (Jiang and Sehityoglu, 1999) and a ratchetting criterion (Kapoor, 1994) was proven successful to predict the fatigue initiation in rails (Ringsberg, 2001). Some 'extended' uniaxial criteria can also be found in the literature, but were proven less efficient (Ringsberg, 2000). More simplified models were developed to associate the fatigue lifetime to some parameters such as the contact pressure. An overview of some predictive models was given by Ekberg et al. (2005) and Stone and Moyar (1989), with interesting discussions on influencing factors such as the impact loading, thermal loading, martensite formation, etc. An elasto-plastic constitutive model considering the damage, isotropic and kinematical hardening was presented by Kunc et al. (2007). Their main 
goal was to determine the charge capacity for low cycle loadings in the raceways of big rolling bearings at low rotation speeds. The model implemented in a finite element code was used to study the evolution of the elasto-plastic strain and stress states, as well as the damage occurrence. Some authors used a linear kinematical hardening model (Gupta et al., 1993; Guo and Barkey, 2004a; Liu et al., 2006) with a limited application field. Others proposed a nonlinear one to improve it (Ringsberg, 2001; Kunc et al., 2007).

The difficulties of the remeshing during the rupture evolution and its great influence on the accuracy of the results were studied by Guo and Barkey (2004b) and Spiteri et al. (2007). The use of a fine element mesh required too much CPU time for the fatigue test simulation (Liu et al., 2006; Busquet et al., 2005; Ringsberg et al., 2005, Li et al., 2006). In fact, the problem of meshing, remeshing and deleting of the damaged element is a crucial point for a fast and accurate fatigue damage simulation.

Our objective is to develop a numerical tool, based on the finite element method, to determine the damage evolution and the remaining lifetime for mechanic parts under cyclic loadings. In this study, we firstly introduce the Sines's fatigue criterion considering the multiaxial stress state to evaluate the appearance and growth of the fatigue damage, then we present the fatigue damage model of Lemaitre and Chaboche based on the continuum damage mechanics and on the thermodynamics of irreversible processes. This damage model allows one to estimate the evolution of the damage value at a material point and its lifetime in terms of number of cycles. The used damage model is coupled with the elasto-plastic behaviors by using an efficient weak coupling procedure. A cycle jumping algorithm is proposed, leading to a large reduction of the CPU time. Some remeshing and element deleting techniques are implemented, which allow one to well follow the crack propagation. The present fatigue damage modeling and numerical techniques are applied to a $2 \mathrm{D}$ specimen under a cyclic stretching. The encouraging results show that they will be very useful tools for the lifetime prediction in the field of maintenance.

\section{FORMULATION ASPECTS OF FATIGUE DAMAGE}

The present fatigue damage model deals with the birth and growth of micro-fissures under cyclic loadings, until the beginning of macroscopic fissures. This phenomenon can occur even if the stress level is inferior to the elastic limit of the material, but the fatigue damage is often coupled with the plasticity. 


\section{Definition of the Damage Variable}

The damage effect on the stresses is given by the following strain-stress relation (Saanouni et al., 2000; Saanouni and Chaboche, 2003):

$$
\underline{\sigma}=(1-D) \underline{\underline{\Lambda}}: \underline{\varepsilon}
$$

where $\underline{\underline{\Lambda}}$ is the elastic constitutive matrix, $\underline{\sigma}$ the stress tensor and $\underline{\varepsilon}$ the strain tensor. When the damage value increases, the effective stiffness modulus $(1-D) \underline{\underline{\Lambda}}$ decreases. After a number of cycles, the critical state may appear in a finite region, where the mechanical integrity and stiffness are completely lost. This area can be considered as the continuum damage representation of a crack.

\section{Fatigue Criteria for 3D Stress States}

To take into account of the multiaxial stress character, a fatigue damage criterion $\left(f_{f}\right)$ similar to the flow surface of plasticity, was proposed by Lemaitre and Chaboche (1990). The fatigue damage occurs when $f_{f}$ is positive:

with

$$
f_{f}=A_{I I}-A_{I I}^{*}
$$

$$
A_{I I}=\frac{1}{2} \sqrt{\frac{3}{2}\left(\underline{\sigma}_{\max }^{\mathrm{dev}}-\underline{\sigma}_{\min }^{\mathrm{dev}}\right):\left(\underline{\sigma}_{\max }^{\mathrm{dev}}-\underline{\sigma}_{\min }^{\mathrm{dev}}\right)}
$$

where $\underline{\sigma}_{\max }^{\mathrm{dev}}$ and $\underline{\sigma}_{\min }^{\mathrm{dev}}$ are the deviatoric tensors of the maximum and minimal stresses in a loading cycle.

In our study, the Sines criterion (Sines and Oghi, 1981) with the damage consideration is adopted:

$$
A_{I I}^{*}=(1-D) \sigma_{l o}\left(1-3 b \bar{\sigma}_{H}\right)
$$

where $\bar{\sigma}_{H}=\operatorname{Av}(\operatorname{Tr}(\underline{\sigma}) / 3)$ is the average hydrostatic stress in a loading cycle, $\sigma_{l o}$ the fatigue limit of an alternative loading $(\bar{\sigma}=0)$ and $b$ a material coefficient.

\section{Evolution Laws of Fatigue Damage}

Under a cyclic loading, the fatigue damage laws for a ID problem depend on the average stress value $(\bar{\sigma})$, the maximal stress $\left(\sigma_{M}\right)$, and the number of cycles $(N)$. 
The following ID fatigue damage model was proposed by Lemaitre and Chaboche (1990):

$$
\delta D=\left[1-(1-D)^{\beta+1}\right]^{\alpha\left(\sigma_{M}, \bar{\sigma}\right)}\left[\frac{\sigma_{M}-\bar{\sigma}}{M(\bar{\sigma})(1-D)}\right]^{\beta} \delta N
$$

with

$$
\begin{aligned}
M(\bar{\sigma}) & =M_{0}(1-b \bar{\sigma}) ; \quad M_{0}=B[a(\beta+1)]^{1 / \beta} \\
\alpha\left(\sigma_{M}, \bar{\sigma}\right) & =1-a\left\langle\frac{\sigma_{M}-\sigma_{l}(\bar{\sigma})}{\sigma_{u}-\sigma_{M}}\right\rangle ; \quad \sigma_{l}(\bar{\sigma})=\sigma_{l_{0}}+\left(1-b \sigma_{l_{0}}\right) \bar{\sigma}
\end{aligned}
$$

where $\sigma_{u}$ is the ultimate stress at the static rupture, the functions $M(\bar{\sigma})$ and $\alpha\left(\sigma_{M}, \bar{\sigma}\right)$ are chosen by considering the stress limit of fatigue, the static rupture and the nonlinear accumulation effects, the material coefficients $M_{0}$, $\beta, B, a$, and $b$ can be determined using a SN experimental curve.

A 3D fatigue damage model can be obtained by using: (a) the 1D law which describes correctly the damage evolution in tension and the effects of nonlinear accumulation; (b) a 3D criterion of fatigue which guides the choice of the significant stress invariants in the law; (c) the anisotropy of the damage behavior.

Thus, the evolution law of fatigue damage for a $3 \mathrm{D}$ stress state is written as follows:

$$
\delta D=\left[1-(1-D)^{\beta+1}\right]^{\alpha}\left(\frac{A_{I I}}{M(1-D)}\right)^{\beta} \delta N
$$

with

$$
\begin{aligned}
M & =M_{0}\left(1-3 b \bar{\sigma}_{H}\right) ; \quad \alpha=1-a\left\langle\frac{A_{I I}-A_{I I}^{*}}{\sigma_{u}-\sigma_{\text {eqmax }}}\right\rangle \text { and } \\
\sigma_{\text {eqmax }} & =\max \left(\sqrt{\frac{3}{2} \underline{\sigma}^{\operatorname{dev}}: \underline{\sigma}^{\operatorname{dev}}}\right) .
\end{aligned}
$$

Supposing that the stress state is saturated and remains quasi-constant, the integration of the Equation (6) can give a rough estimation of the damage at the cycle $N$ :

$$
D_{N+\Delta N}=1-\left\{1-\left[\frac{\Delta N}{N_{f}}+\left[1-\left(1-D_{N}\right)^{\beta+1}\right]^{-\alpha+1}\right]^{1 / 1-\alpha}\right\}^{1 / \beta+1}
$$

with

$$
N_{f}=\frac{1}{(\beta+1)(1-\alpha)}\left(\frac{A_{I I}}{M}\right)^{-\beta}
$$


But generally, the stress state is not constant due to the coupling of the fatigue damage and elasto-plastic behaviors. The estimate of the damage is done in an incremental form:

$$
D_{N+\Delta N}=D_{N}+\Delta D
$$

where $D_{N}$ is the known damage value at the cycle $N, \Delta D$ is the damage increment corresponding to the increment of the number of cycles $\Delta N$.

The above calculation implemented in our routine Vumat (ABAQUS, 2006) is very fast since the plasticity and damage are computed separately in an explicit form (weak coupling).

\section{Internal Variables and Constitutive Equations}

In the present model, the fatigue damage is coupled with the elasto-plastic behaviors. In the constitutive equations, the following variables are used:

- $\left(\underline{\sigma}, \dot{\varepsilon}^{\mathrm{p}}\right)$ : the Cauchy stress tensor and plastic strain rate tensor to describe the plastic flow,

- $(\underline{X}, \underline{\dot{\alpha}})$ : the deviatoric tensors to describe the kinematical hardening,

- $(R, \dot{r})$ : the scalar variables to describe the isotropic hardening.

The coupled constitutive equations are derived from the state and dissipation potentials using the generalized normality rule based on the nonassociative theory (Saanouni et al., 2000).

The yield function of plasticity with the damage consideration is given by Lemaitre and Chaboche (1990):

$$
f_{p}=\frac{J_{2}(\underline{\sigma}-\underline{X})}{\sqrt{1-D^{*}}}-\frac{R}{\sqrt{1-D^{*}}}-\sigma_{y}=0
$$

with

$$
J_{2}(\underline{\sigma}-\underline{X})=\sqrt{\frac{3}{2}(\underline{\sigma}-\underline{X}):(\underline{\sigma}-\underline{X})}
$$

The stress tensor and plastic strain rate tensor are calculated by the following equations:

$$
\left\{\begin{array}{l}
\underline{\sigma}=\left(1-D^{*}\right)\left[\lambda_{e}\left(\underline{\varepsilon}^{e}: \underline{1}\right) 1+2 \mu \underline{\varepsilon}^{e}\right] \\
\dot{\varepsilon}^{p}=\dot{\lambda} \frac{3}{2} \frac{1}{\sqrt{1-D^{*}}} \frac{\underline{\sigma}^{\operatorname{dev}}-\underline{X}}{J_{2}(\underline{\sigma}-\underline{X})}=\dot{\lambda} \underline{n}
\end{array}\right.
$$

where $\lambda$ and $\mu$ are the Lamé's coefficients, the damage value at the previous cycle $D^{*}$ is kept constant during the computation of plasticity, this weak 
coupling between the mechanical behavior and damage allows to speed up the computation. The coupling effect is realized with the term $\left(1-D^{*}\right)$; the uncoupling calculation with $D^{*}=0$ in the above equations allows to study the coupling influence on the lifetime prediction (Figure 4(b)).

The well-known Return Mapping Method proposed by Simo and Ortiz (1985) is used in this study to integrate the coupled damage-plasticity equations. This method uses an elastic prediction followed by a plastic correction and an implicit resolution scheme. There is only a single yielding surface for the coupled damage-plasticity behaviors. The details related to this aspect can be found in Saanouni et al. (2000), where three different iterative implicit schemes were discussed and compared for the fully coupled constitutive equations including the elasto-plasticity, anisotropy, damage, and kinematical hardening.

An efficient explicit algorithm is used considering a weak coupling between the damage and plasticity. At a time increment, we use the damage value obtained at the previous increment to calculate the stress tensor in the plastic integration procedure; then we use the obtained stress tensor to determine the new damage value; the influence of the new damage value on the stress state will be considered in the next time increment (Lestriez et al., 2004). Thus, the Mises criterion can be expressed only in function of the plastic multiplier $\Delta \lambda$ and solved by Simo's classical return mapping method.

\section{NUMERICAL ASPECTS AND IMPLEMENTATION}

\section{Cycle Jumping Algorithm}

It is too time-consuming to simulate every loading cycle to estimate the damage evolution. A cycle jumping method should be used to speed up the calculation. At the fatigue damage stage, the cycle jumping increment $\Delta N$ can be easily determined by Equation (6) for a given damage increment $\Delta D$. But this $\Delta D$ may lead to a very great jumping before and at the beginning of the fatigue damage. So, a calculation without damage consideration is used to stabilize the stress state $\left(A_{I I}\right)$ due to the plastic hardening effects.

A cycle jumping algorithm is proposed to rapidly attain the stabilized state. At the beginning, the elasto-plastic calculations are carried out without damage effects. The cycle jumping increment $\Delta N$ is determined according to the gradient of an internal variable such as the stress state. In the case of elastic fatigue, several increments are sufficient to reach a stable stress state (once $\Delta N>$ the imposed cycle increment threshold $\Delta N s$ ); in the case of plastic fatigue, numerous increments are needed to stabilize the stress state due to the plastic hardening. This stabilization is very important 
for the determination of the state stress $A_{I I}$ in order to better calculate the cycle jumping increment $\Delta N$ and the fatigue damage.

Firstly, the internal variable $y(t)$ in function of time is transformed into a function of number of cycles $y(N)$; then this variable at the cycle $N+\Delta N$ can be developed into a Taylor series of second order:

$$
y_{N+\Delta N}=y_{N}+\dot{y}_{N} \Delta N+\ddot{y}_{N} \frac{\Delta N^{2}}{2}+\cdots
$$

In our case, the term of second order is very small compared to other terms, so negligible:

$$
\left|\dot{y}_{N}\right| \Delta N \approx\left|y_{N+\Delta N}-y_{N}\right| \leq \eta\left|y_{N}\right|
$$

where $\eta$ is a positive coefficient to adjust the jumping increment size.

The derivative $\dot{y}_{N}$ can be written by using the finite difference method between $y_{N}$ and $Y_{N+1}$. Thus, we obtain a criterion to determine the jumping increment $\Delta N$ :

$$
\dot{y}_{N}=\frac{y_{N+1}-y_{N}}{1} \quad \text { and } \quad \Delta N \leq \eta\left|\frac{y_{N}}{y_{N+1}-y_{N}}\right|
$$

This cycle jumping algorithm allows one to largely reduce calculation times to attain a stabilized stress state for the following fatigue damage simulation. It is advisable to calculate the cycle jumping size for each internal variable and select the minimal one. Some numerical trials also showed that it is better to take the maximal stress as internal variable instead of the cumulative plastic strain.

Once a stable stress state is attained, the damage should be taken into account and the cycle jumping increment $\Delta N$ is determined by Equation (6) for a given damage increment ( $\Delta D=0.025$ for example).

\section{REMESHING TECHNIQUES FOR DAMAGE SIMULATION}

In order to better follow the crack propagation, the finite element mesh should be adapted to the damage evolution. This adaptative remeshing is performed only during the softening stage where the damage attains a high value and leads to a crack birth and growth. The remeshing procedure is carried out as follows:

- Determination of remeshing zones: the damage value $D$ is examined on the whole structure, the entirely damaged elements and 'free' elements are deleted, the partially damaged elements are included in a remeshing zone. 
- Specification of the mesh density: a coefficient of element size variation (currently set to 1.2) is used to ensure the small size variation between adjacent elements.

- Generation of the boundary nodes, interior nodes and elements: starting with the boundary nodes, the interior nodes and triangle elements are generated by using the advancing-front technique; then a mesh improving process is used to optimize the mesh quality.

- Transfer of state variables to the new mesh by a weighted interpolation technique.

\section{NUMERICAL RESULTS, APPLICATION ON A 2D SPECIMEN}

The present fatigue damage model is applied to a $2 \mathrm{D}$ specimen (Figure 1(a)). The used material is the 316L stainless steel. A cyclic loading in extension-compression is given by an imposed cyclic displacement.

(a)

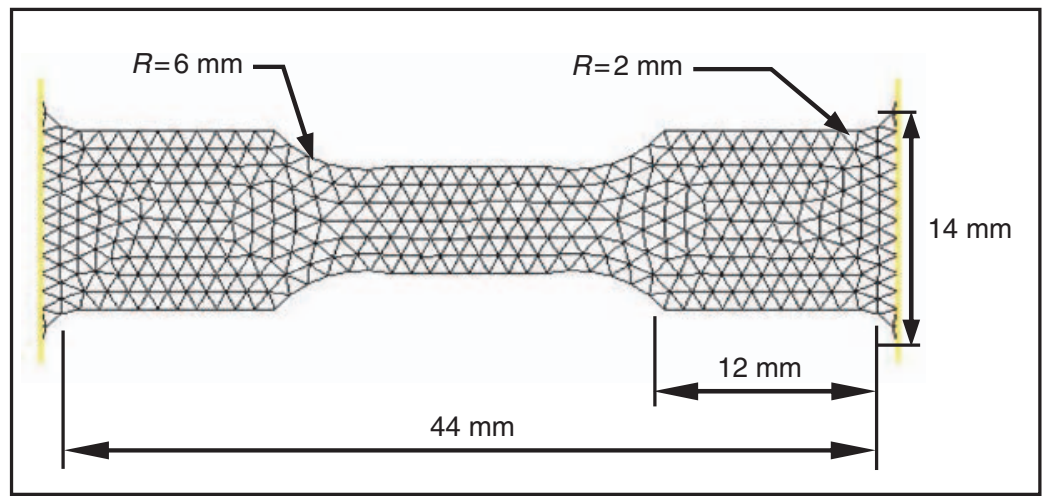

(b)

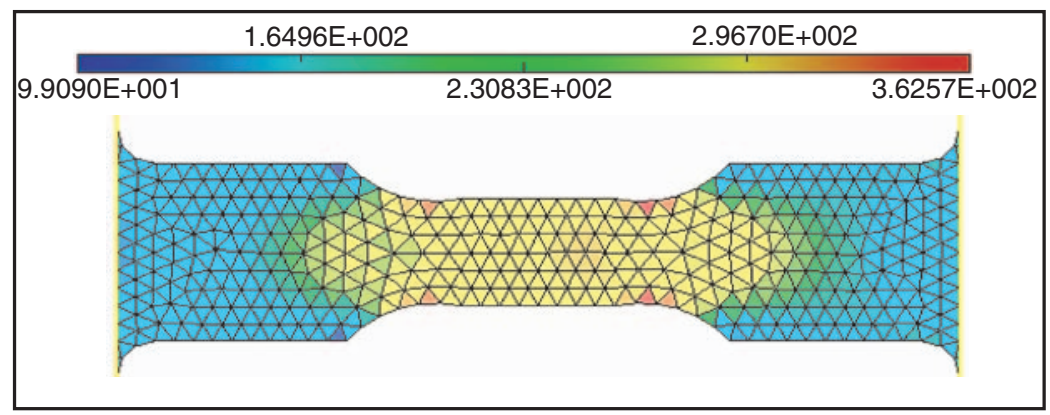

Figure 1. (a) Tension fatigue test specimen, (b) Distribution of Von Mises stress with cycle jumping. 

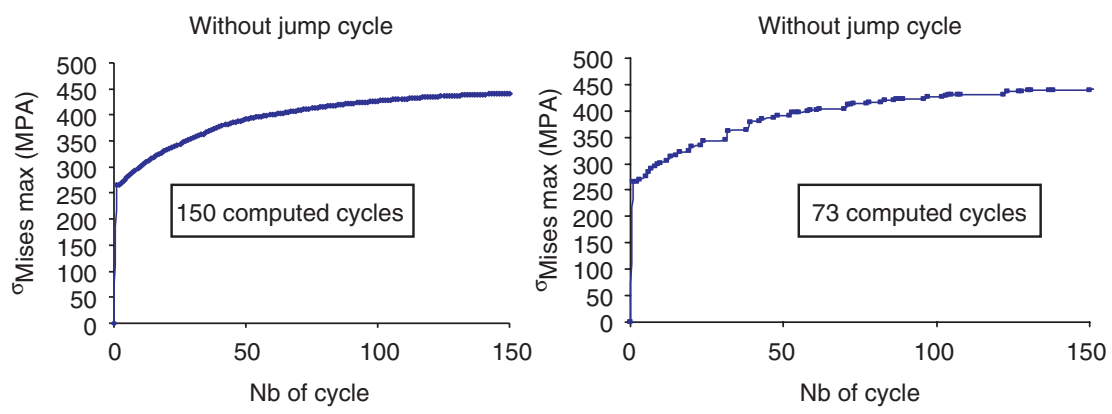

Figure 2. Simulations with or without cycle jumping algorithm.

The specimen is discretized with the Constant Strain Triangular element CPE3 in ABAQUS library.

The fatigue simulation is carried out with or without the cycle jumping algorithm. Very similar curves are obtained (Figure 2), but the cycle jumping algorithm allows one to use only 73 increments instead of 150 ones.

The stress distribution is shown in Figure 1(b). Although the number of increments is largely reduced using the cycle jumping algorithm, the Von Mises stress value and its symmetrical distribution are still in good agreement with those obtained without cycle jumping. Several calculations are carried out to study the cyclic force response versus the number of cycles (Figure 3). We note that the amplitude of the imposed cyclic displacement $u$ strongly influences the lifetime of the specimen, very similar phenomenon can be found in the literature (Alain et al., 1997, Lim et al., 2005) for this kind of stainless steel material. This figure clearly shows the efficiency of the cycle jumping algorithm: for instance, in the case of the small imposed displacement $u=0.0375 \mathrm{~mm}$, only 750 increments are used for 51,000 cycles.

We can also observe different stages of the material resistance. All curves illustrate three stages: a stress increase stage due to the hardening, a damage growth stage (more or less long) and an entire damage stage leading to the total rupture and force falling. This is the typical behavior of the austenitic steel $316 \mathrm{~L}$.

The initiation and evolution of the crack until the total rupture of the specimen are shown in Figure 4. We note that the total rupture of the specimen is quickly attained after the crack initiation (only 26 cycles) in the case of the imposed cyclic displacement $u=0.075 \mathrm{~mm}$.

It is interesting to observe the mesh refinement in these figures. The isocolor values represent the damage distribution $(D=0$ means healthy elements, $D=1$ means entire damaged elements). The mesh of the healthy 


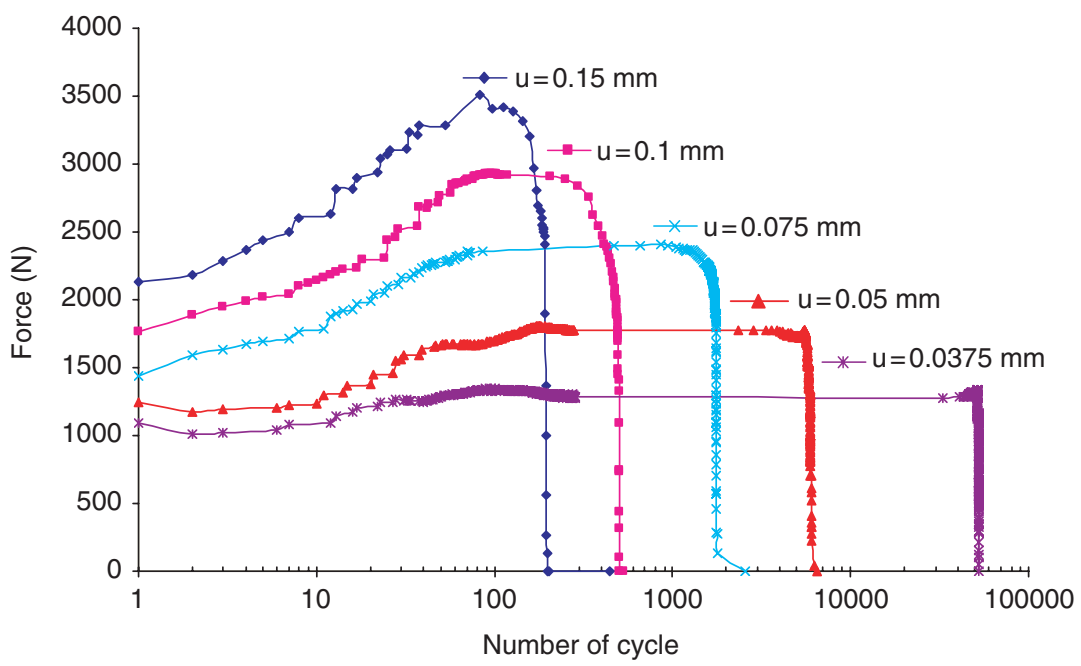

Figure 3. Force vs number of cycles for various imposed cyclic stretching.
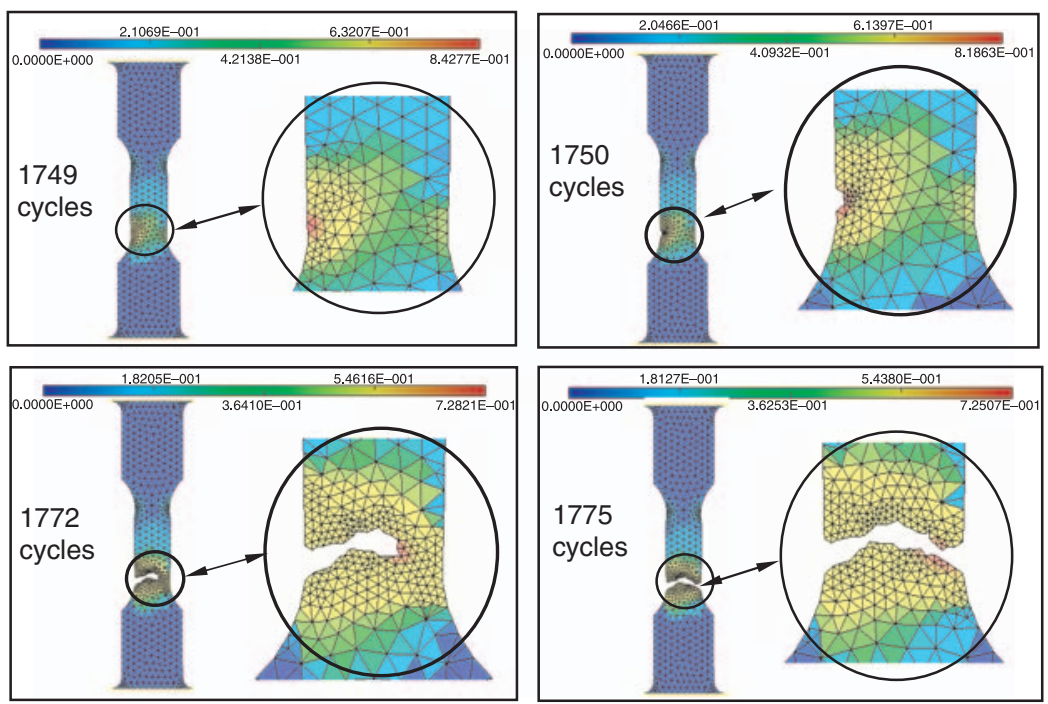

Figure 4. Rupture evolution due to the fatigue damage.

zones remains unchanged, the entirely damaged elements and 'cutting' elements have been deleted, the partially damaged zones have been remeshed. We note that the mesh refinement well follows the damage evolution and illustrates the rupture propagation. 


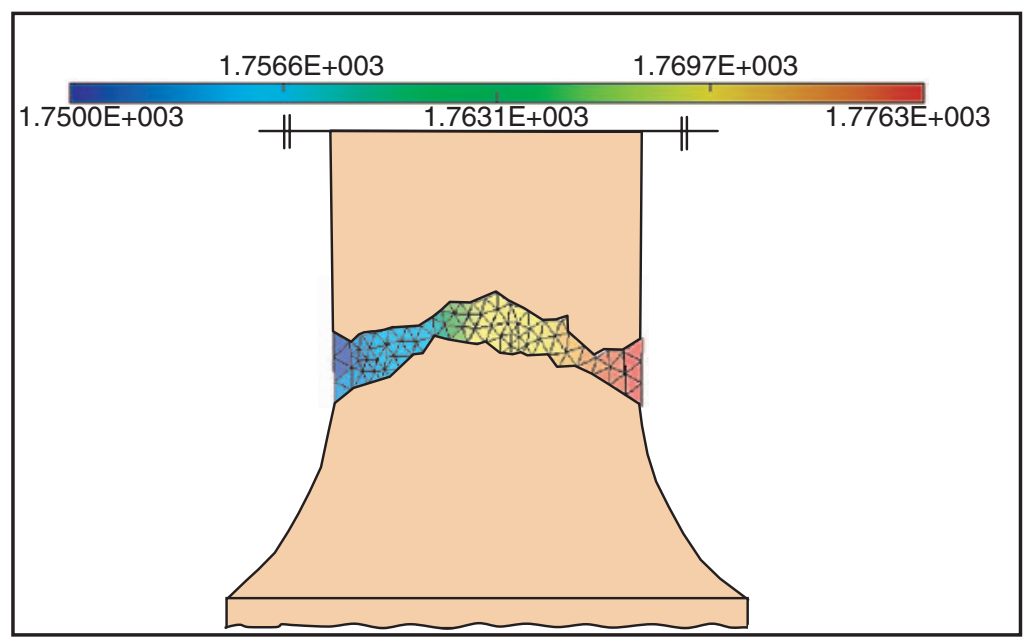

Figure 5. Number of cycles needed for each damage element.

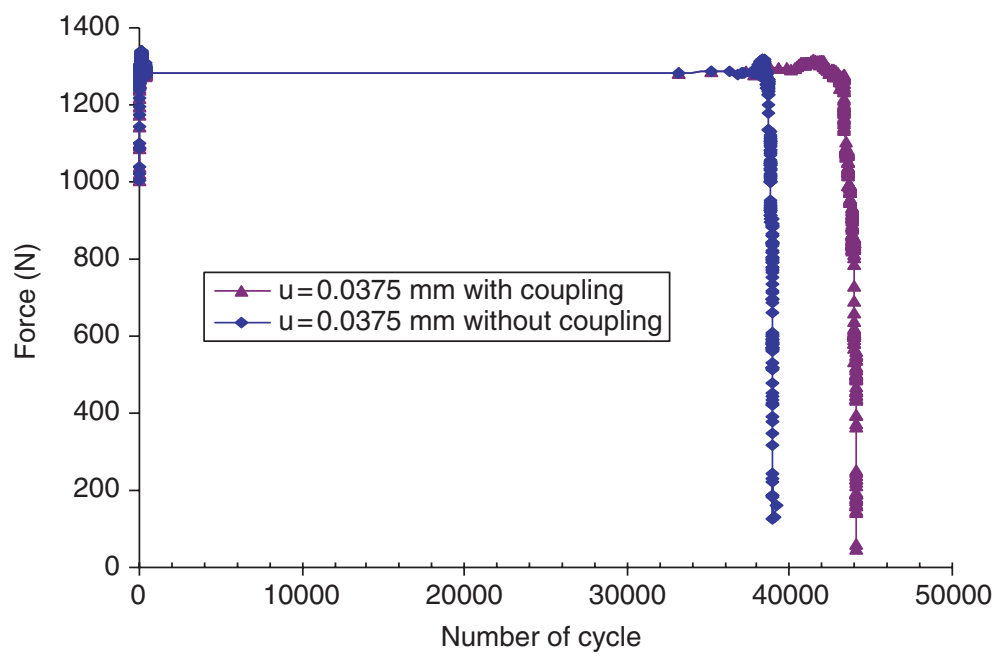

Figure 6. Influence of the coupling effect on the lifetime of the specimen.

Figure 5 shows the number of cycles needed to entirely damage an element. Obviously, we can also know the number of cycles needed for the initiation and evolution of the rupture in the part.

The coupling effect between the damage and elasto-plastic behaviors has a great influence on the lifetime of a part under a cyclic loading. The Figure 6 shows the evolution of the damage (or loss of the resistance of material) 
obtained by the calculations with or without the coupling effect. The coupling effect is realized with the term $\left(1-D^{*}\right)$; the uncoupling calculation with $D^{*}=0$ in the constitutive equations (9) and (10) allows to study the coupling influence on the lifetime prediction.

We can observe that the coupling effect is notable on the lifetime (more than $12 \%$ ). This remark is very important for the prediction of the remaining lifetime of a mechanical part such as a chipped ball bearing.

\section{CONCLUSION AND PERSPECTIVES}

A fatigue damage model based on the continuum damage mechanics is developed to determine the damage evolution and the lifetime of mechanical parts under cyclic loading. The Sines fatigue criterion is adopted and modified to take into account the coupling of the damage and plasticity. The importance of the coupling effect for the determination of the remaining lifetime is shown by a comparison of the results obtained with or without the coupling. A cycle jumping algorithm is proposed which leads to a considerable CPU time saving. Some remeshing techniques allow one to delete the damaged elements and follow the rupture propagation with good refined meshes.

The present model is applied to a $2 \mathrm{D}$ specimen under a cyclic stretching. The comparison of the results with those in the literature gives a qualitatively good agreement.

Our recent numerical trials to simulate the chipping initiation and evolution of a bearing thrust give interesting results. Some improvements concerning the residual stress and the surface roughness seem important for this kind of problems. A quadrilateral element with the corresponding remesher will also be developed to obtain more stable behaviors relative to the hydrostatic stress.

Other future works will be to characterize the fatigue behaviors of different materials for the simulation data. The experimental validation of the specimen in cyclic stretching will be carried out.

\section{REFERENCES}

ABAQUS (2006). Documentation v6.6.

Alain, R., Violan, P. and Mendez, J. (1997). Low Cycle Fatigue Behaviour in Vacuum of a 316L Type Austenitic Stainless Steel Between 20 and $600^{\circ} \mathrm{C}$, Part I: Fatigue Resistance and Cyclic Behaviour, Mater. Sci. Eng., A 229: 87-94.

Bogard, F., Debray, K. and Guo, Y.Q. (2002). Determination of Sensor Positions for Predictive Maintenance of Revolving Machines, Int. J. Solids and Structures, 39: 3159-3173. 
Busquet, M., Baillet, L., Bordreuil, C. and Berthier, Y. (2005). 3D Finite Element Investigation on the Plastic Flows of Rolling Contacts - Correlation with Rail Head Microstructural Observations, Wear, 258: 1071-1080.

Chaboche, J.L. (1974). Une loi Différentielle D'endommagement de Fatigue avec Cumulation Non linéaire, Revue Française de Mécanique, No. 50-51: 71-82.

Ekberg, A. and Kabo, E. (2005). Fatigue of Railway Wheels and Rails Under Rolling Contact and Thermal Loading-an Overview, Wear, 258(7-8): 1288-1300.

Guo, Y.B. and Barkey, M.E. (2004a). Modelling of Rolling Contact Fatigue for Hard Machined Components with Process-induced Residual Stress, Int. J. Fatigue, 26: 605-613.

Guo, Y.B. and Barkey, M.E. (2004b). FE-simulation of the Effects of Machining-induced Residual Stress Profile on Rolling Contact of Hard Machined Components, Int. J. Mech. Sci., 46: 371-388.

Gupta, V., Bastias, P., Hahn, G.T. and Rubin, C.A. (1993). Elasto-plastic Finite-element Analysis of 2-D Rolling Sliding Contact with Temperature-dependent Bearing Steel Material Properties, Wear, 169: 251-256.

Jiang, Y. and Sehitoglu, H. (1999). A Model for Rolling Contact Fatigue, Wear, 224: 38-49.

Kachanov, L.M. (1986). Introduction to Continuum Damage Mechanic, Martinus Nijhof, Netherlands.

Kapoor, A. (1994). A Re-evaluation of the Life to Rupture of Ductile Metals by Cyclic Plastic Strain, Fatigue Fract. Eng. Mater. Struct., 17: 201-219.

Kunc, R., Zerovnik, A. and Prebil, I. (2007). Verification of Numerical Determination of Carrying Capacity of Large Rolling Bearings with Hardened Raceway, Int. J. Fatigue, 29(9-11): 1913-1919.

Lemaitre, J. and Chaboche, J.L. (1990). Mechanics of Solid Materials (Trans. B. Shrivastava), Cambridge University Press, Cambridge, UK.

Lestriez, P., Saanouni, K., Mariage, J.F. and Cherouat, A. (2004). Numerical Prediction of Damage in Metal Forming Process Including Thermal Effects, Int. J. Damage Mec., 13(1): $59-80$.

Li, B., Reis, L. and De Freitas, M. (2006). Simulation of Cyclic Stress/Strain Evolutions for Multiaxial Fatigue Life Prediction, Int. J. Fatigue, 28: 451-458.

Lim, J.Y., Hong, S.G. and Lee, S.B. (2005). Application of Local Stress-Strain Approaches in the Prediction of Fatigue Crack Initiation Life for Cyclically Non-Stabilized and Non-Massing Steel, Int. J. Fatigue, 27(10-12): 1653-1660.

Liu, Y., Stratman, B. and Mahadevan, S. (2006). Fatigue Crack Initiation Life Prediction of Railroad Wheels, Int. J. Fatigue, 28: 747-756.

Rabotnov, Y.N. (1969). Creep Problems in Structural Members, North-Holland, Amsterdam.

Ringsberg, J.W. (2000). Rolling Contact Fatigue of Railway Rails with Emphasis on Crack Initiation, PhD Thesis, Dep. of Solid Mechanics, Chalmers University of Technology, Göteborg, Sweden.

Ringsberg, J.W. (2001). Life Prediction of Rolling Contact Fatigue Crack Initiation, Int. J. Fatigue, 23: 575-586.

Ringsberg, J.W., Franklin, J., Josefson, B., Kapoor, A. and Nielsen, C.O. (2005). Fatigue Evaluation of Surface Coated Railway Rails Using Shakedown Theory, Finite Element Calculations and Lab and Field Trials, Int. J. Fatigue, 27: 680-694.

Saanouni, K. and Chaboche, J.L. (2003). Computational Damage Mechanics. Application to Metal Forming, Numerical and Computational Methods, R. de Borst and H. A. Mang (eds), In: Comprehensive Structural Integrity, Chapter 7, Vol. 3, ISBN: 0-08-043749-4, Elsevier, UK.

Saanouni, K, Nesnas, K. and Hammi, Y. (2000). Damage Modelling in Metal Forming Processes, Int. J. Damage Mech., 9(3): 196-240. 
Sines, G. and Ohgi, G. (1981). Fatigue Criteria Under Combined Stresses or Strains, Journal of Engineering Materials and Technology, 103: 82-90.

Simo, J.C. and Ortiz, M. (1985). A Unified Approach to Finite Deformation Elastoplastic Analysis Based on the use of Hyperelastic Constitutive Equations, Comp. Meth. Appl. Eng., 51: 221-245.

Spiteri, P., Ho, S. and Lee, Y. L. (2007). Assessment of Bending Fatigue Limit for Crankshaft Sections with Inclusion of Residual Stresses, Int. J. Fatigue, 29: 318-329.

Stone, D.H. and Moyar, G.J. (1989). Wheel Shelling and Spalling - an Interpretive Review, In: Rail Transportation, pp. 19-31, ASME. 